\title{
Dissociation of Activated Protein C Functions by Elimination of Protein S Cofactor Enhancement ${ }^{*}$
}

Received for publication, March 25, 2008, and in revised form, August 27, 2008 Published, JBC Papers in Press, September 8, 2008, DOI 10.1074/jbc.M802338200

\author{
Shona Harmon ${ }^{\ddagger 1}$, Roger J. S. Preston ${ }^{\ddagger 1,2}$, Fionnuala Ni Ainle ${ }^{\ddagger}$, Jennifer A. Johnson ${ }^{\ddagger}$, Moya S. Cunningham $^{\ddagger}$, \\ Owen P. Smith ${ }^{\S}$, Barry White", and James S. O’Donnell ${ }^{\mp \uparrow}$ \\ From the ${ }^{\ddagger}$ Haemostasis Research Group, Institute of Molecular Medicine, St James's Hospital, Trinity College Dublin, Dublin 8, the \\ ${ }^{\S}$ Department of Haematology, Our Lady's Children's Hospital Crumlin, Dublin 12, and the "National Centre for Hereditary \\ Coagulation Disorders, St James's Hospital, James St., Dublin 8, Ireland
}

Activated protein $\mathrm{C}$ (APC) plays a critical anticoagulant role in vivo by inactivating procoagulant factor $\mathrm{Va}$ and factor VIIIa and thus down-regulating thrombin generation. In addition, APC bound to the endothelial cell protein $C$ receptor can initiate protease-activated receptor-1 (PAR-1)-mediated cytoprotective signaling. Protein $S$ constitutes a critical cofactor for the anticoagulant function of APC but is not known to be involved in regulating APC-mediated protective PAR-1 signaling. In this study we utilized a site-directed mutagenesis strategy to characterize a putative protein $S$ binding region within the APC Gla domain. Three single amino acid substitutions within the APC Gla domain (D35T, D36A, and A39V) were found to mildly impair protein $\mathrm{S}$-dependent anticoagulant activity (<2-fold) but retained entirely normal cytoprotective activity. However, a single amino acid substitution (L38D) ablated the ability of protein $\mathrm{S}$ to function as a cofactor for this APC variant. Consequently, in assays of protein $\mathrm{S}$-dependent factor Va proteolysis using purified proteins or in the plasma milieu, $A P C-L 38 D$ variant exhibited minimal residual anticoagulant activity compared with wild type APC. Despite the location of Leu-38 in the Gla domain, APC-L38D interacted normally with endothelial cell protein $C$ receptor and retained its ability to trigger PAR-1 mediated cytoprotective signaling in a manner indistinguishable from that of wild type APC. Consequently, elimination of protein $S$ cofactor enhancement of APC anticoagulant function represents a novel and effective strategy by which to separate the anticoagulant and cytoprotective functions of APC for potential therapeutic gain.

Protein $\mathrm{C}$ is a vitamin $\mathrm{K}$-dependent serine protease zymogen that circulates at a plasma concentration of $\sim 70 \mathrm{nM}$. It has a multidomain structure comprising an $\mathrm{N}$-terminal $\gamma$-carboxyglutamic acid $(\mathrm{Gla})^{3}$ domain (residues $\left.1-45\right)$, two epidermal growth factor-

* This work was supported by a Health Research Board Fellowship PD/2006/24 (to R. J. S. P.), an Irish Heart Foundation grant (to J. S. O. D.), a Children's Medical Research Foundation Award (to F. N. A. and O. P. S.), and Science Foundation Ireland President of Ireland Young Researcher Award 06/Y12/0925 (to J.S. O. D.). The costs of publication of this article were defrayed in part by the payment of page charges. This article must therefore be hereby marked "advertisement" in accordance with 18 U.S.C. Section 1734 solely to indicate this fact.

${ }^{1}$ These authors contributed equally to this study.

${ }^{2}$ To whom correspondence should be addressed. Tel.: 353-1-896-3274; Fax: 353-1-410-3570; E-mail address: prestonr@tcd.ie.

${ }^{3}$ The abbreviations used are: Gla, $\gamma$-carboxyglutamic acid; APC, activated protein $C$; EPCR, endothelial cell protein $C$ receptor; SEPCR, soluble EPCR; Fva, factor Va; PC, phosphatidylcholine; PE, phosphatidylethanolamine; PS, like domains (EGF-1, residues 46-92, and EGF-2, residues 93-136) and a C-terminal serine protease domain (170-419). Protein $\mathrm{C}$ zymogen is activated by thrombin in complex with thrombomodulin on the surface of endothelial cells (1). Activated protein $C$ (APC) generation is significantly enhanced by protein $C$ binding to the endothelial cell protein $C$ receptor (EPCR) (2). APC binds to anionic phospholipids on the endothelial cell surface and, in complex with its cofactor protein $\mathrm{S}$ inactivates procoagulant cofactors factor Va (FVa) (3) and factor VIIIa (4), thereby attenuating further thrombin generation and down-regulating coagulation. The physiological importance of the protein $\mathrm{C}$ anticoagulant pathway is well established. Individuals with homozygous protein $\mathrm{C}$ deficiency typically present shortly after birth with fulminant life-threatening thrombotic complications (5). Moreover, individuals heterozygous for protein $\mathrm{C}$ deficiency demonstrate significant lifelong increased risk of venous thromboembolism (6).

Recent studies have shown that APC bound to EPCR via its Gla domain can activate protease-activated receptor-1 (PAR-1) on endothelial cells, triggering complex intracellular signaling (7). The molecular mechanisms underlying the cytoprotective effects of APC have not been fully characterized but appear mediated independently of its anticoagulant function (8). Through mechanisms downstream of PAR-1 activation, APC has been shown to exhibit anti-inflammatory and anti-apoptotic properties via up-regulated gene expression of anti-apoptotic and anti-inflammatory mediators $(9,10)$, down-regulation of pro-inflammatory cytokines $(10,11)$, and stabilization of endothelial barrier function $(12,13)$.

In vivo beneficial effects of APC have been reported in a number of different animal injury models. For example, APC demonstrated significant neuroprotective effects in rat and murine stroke models $(14-16)$ and was also recently shown to ameliorate experimental autoimmune encephalomyelitis in mice (17). Furthermore, APC was protective in a murine model of diabetic nephropathy by inhibition of endothelial and podocyte apoptosis, whereas anticoagulation with low molecular weight heparin had no such beneficial effect (18). Each of these animal studies demonstrated a consistent and critical role for the APC-EPCRPAR-1 signaling axis.

The therapeutic potential of APC was highlighted in the activated Protein C Worldwide Evaluation in Severe Sepsis

phosphatidylserine; ETP, endogenous thrombin potential; PAR-1, protease-activated receptor-1; PROWESS, activated Protein C Worldwide Evaluation in Severe Sepsis. 
(PROWESS) study (19). Intravenous infusion of recombinant APC $(24 \mu \mathrm{g} / \mathrm{kg}$ per $\mathrm{h}$ for $96 \mathrm{~h})$ significantly reduced overall mortality (relative risk reduction, 19.4\%) in adult patients with severe sepsis. In contrast, treatment with other anticoagulants (notably antithrombin (20) and tissue factor pathway inhibitor (21)) have no such effect on survival. The anticoagulant properties of APC are, however, associated with increased risk of bleeding complications and have thereby restricted its use in clinical practice. In the PROWESS study, serious bleeding complications were observed in $3.5 \%$ of the patients treated with APC compared with only $2 \%$ of the placebo group (19). Furthermore, recent post-marketing studies have reported even higher incidences of serious bleeding complications (6.5 and 6.7\%, respectively) in patients treated with $\operatorname{APC}(22,23)$. In this context, recent studies have sought to define the relative contributions of the cytoprotective and/or anticoagulant properties of APC toward mediating its therapeutic efficacy in different pathological settings. These studies have sought to design APC variants which retain beneficial cytoprotective actions but exhibit relative reduction in its anticoagulant properties and, thus, bleeding risk (24-26).

The anticoagulant function of APC requires the presence of its principal cofactor, protein S (27). Protein S may enhance APC anticoagulant activity by increasing its affinity for negatively charged phospholipid surfaces (27), re-positioning the APC active site for optimal substrate cleavage (28), or removing the protection of FVa in the prothrombinase complex conferred by FXa binding (29). Thus, protein S plays a critical role in mediating the anticoagulant properties of APC, but there is no evidence to date that it is important for the anti-inflammatory or anti-apoptotic effects of APC. The molecular basis through which protein $\mathrm{S}$ interacts with APC is not fully understood; however, a possible role for the APC Gla domain has recently been described (30). In this study, we have used a sitedirected mutagenesis strategy to characterize the impact of individual amino acid residue substitutions in this putative protein $\mathrm{S}$ binding region upon APC anticoagulant function. We demonstrate that, surprisingly, a single amino acid substitution (L38D) is sufficient to entirely ablate the ability of protein $\mathrm{S}$ to function as a cofactor for this APC variant yet does not alter APC-mediated proteolysis of FVa in the absence of protein S. Consequently, in both protein S-dependent FVa proteolysis assays using purified coagulation proteins and in the plasma milieu, this APC Gla domain variant exhibited minimal residual anticoagulant activity compared with wild type APC. In addition, despite the fact that this key amino acid is located in the APC Gla domain, we also show that this APC variant interacts normally with EPCR and that it retains its ability to initiate PAR-1 protective signaling in an identical fashion to that of wild type APC.

\section{EXPERIMENTAL PROCEDURES}

Generation and Characterization of Recombinant Protein $C$ Variants-Recombinant protein C Gla domain variants were generated by site-directed mutagenesis, expressed, and isolated from serum-free medium as previously described (30, 31). Recombinant protein $\mathrm{C}$ was characterized by Coomassie staining and immunodetection using a horseradish peroxidase-con- jugated anti-protein $\mathrm{C}$ polyclonal antibody (Dako, Ely) and a mouse anti-Gla monoclonal antibody (American Diagnostica) according to standard procedures. To generate recombinant APC, wild type protein $C$ and protein $C$ variants were activated with Protac (Hyphen-BioMed). APC chromogenic substrate (BIOPHEN CS-21(66), Hyphen-Biomed) cleavage by each recombinant APC preparation was determined as previously described (30, 31).

Inhibition of APC in Plasma-Inhibition of APC variants in plasma was determined as previously described (32). Wild type and variant APC (20 nM) was incubated in pooled human citrated plasma at $37^{\circ} \mathrm{C}$. At designated time points, aliquots were removed, and the residual amidolytic activity was determined using a chromogenic substrate for APC (BIOPHEN CS2166). To examine the role of protein $C$ inhibitor, the same experiments were performed in the presence of heparin (10 units $/ \mathrm{ml})$.

Determination of APC Anticoagulant Activity in Protein C-deficient Plasma-The anticoagulant function of wild type and variant APC upon protein C-deficient plasma was assessed using a Fluoroskan Ascent Plate Reader (Thermo Lab System, Helsinki, Finland) in combination with Thrombinoscope software (SYNAPSE BV) as previously described (30). $80 \mu$ l of protein C-deficient plasma (Hyphen-Biomed) was incubated with $20 \mu \mathrm{l}$ of PPP reagent (Synapse) containing 5 pm tissue factor and $4 \mu \mathrm{M}$ phospholipids (PC/PS/PE, 40\%:20\%:20\%) in the presence or absence of wild type or variant APC (1.25-20 nM). Thrombin generation was initiated by automatic dispensation of fluorogenic thrombin substrate (Z-Gly-Gly-Arg-amidomethylcoumarin- $\mathrm{HCl}$ ) and $100 \mathrm{~mm} \mathrm{CaCl}$ into each well. Thrombin generation was determined using thrombin calibration standard (Synapse). Measurements were taken at 20-s intervals for 60 min or until thrombin generation was complete. The endogenous thrombin potential (ETP) of each reaction were then calculated. Experiments were performed in triplicate, and data are reported as the mean ETP \pm S.E.

Protein SEnhancement of APC Anticoagulant Activity in Protein S-deficient Plasma-The enhancement of APC anticoagulant activity in protein $\mathrm{S}$-deficient plasma was determined using a similar assay to that described above. Briefly, protein S-deficient plasma (Hyphen-Biomed) was incubated with wild type or variant recombinant $\mathrm{APC}(10 \mathrm{nM})$ in the presence or absence of plasma-purified protein S (0.125-1.5 $\mu \mathrm{M}$, Hematologic Technologies Inc.). Thrombin generation was initiated and assessed using Thrombinoscope software as described previously. All experiments were performed in triplicate and data plotted as mean ETP \pm S.E.

Determination of Protein S-independent APC-mediated Factor Va Proteolysis-FVa degradation by APC was assessed as previously described (30, 31). $0.32 \mathrm{~nm}$ APC was incubated at $37{ }^{\circ} \mathrm{C}$ with phospholipids vesicles (PC/PS/PE, 60\%:20\%:20\%; Avanti Lipids) and 4 nM FVa (Hematologic Technologies, Inc.) in $40 \mathrm{~mm}$ Tris- $\mathrm{HCl}, 140 \mathrm{~mm} \mathrm{NaCl}, 3 \mathrm{~mm} \mathrm{CaCl}_{2}$, and $0.3 \% \mathrm{w} / \mathrm{v}$ bovine serum albumin ( $0.08 \mathrm{nM}$ APC, $19 \mu \mathrm{M}$ phospholipids, and 1 nм FVa, final concentration). Phospholipid vesicles were prepared as described previously (31). At the specified time points, 2 - $\mu$ l aliquots were removed and added to a prothrombinase mixture (25 $\mu \mathrm{M}$ phospholipids, $1 \mathrm{~nm}$ factor Xa, and $0.5 \mu \mathrm{M}$ prothrombin (Hematologic Technologies Inc.) final concentra- 
tions) for $3 \mathrm{~min}$. Each reaction was then stopped using $5 \mu \mathrm{l}$ of ice-cold $0.5 \mathrm{M}$ EDTA. $100 \mu \mathrm{l}$ of the reaction mixture was removed and incubated with $100 \mu \mathrm{l}$ of $2 \mathrm{~mm}$ thrombin chromogenic substrate Biophen CS-01(38) (Hyphen-Biomed) to assess thrombin generation. The rate of chromogenic substrate cleavage was measured at $405 \mathrm{~nm}$ using a plate reader. All experiments were performed in triplicate.

Determination of Protein S-enhanced Proteolysis of FVa by $A P C$-Protein S-enhanced proteolysis of FVa by APC was determined as previously described (30). Human protein S (2.5-25 nM) was incubated with $0.8 \mathrm{nM}$ wild type or variant $\mathrm{APC}, 8 \mathrm{~nm} \mathrm{FVa}$, and $75 \mu \mathrm{M}$ phospholipids vesicles in $40 \mathrm{~mm}$ Tris $\mathrm{HCl}$, pH7.4, $140 \mathrm{~mm} \mathrm{NaCl}, 3 \mathrm{~mm} \mathrm{CaCl}_{2}, 0.3 \%$ (w/v) bovine serum albumin (0.2 nM APC, $2 \mathrm{~nm} F V a$, and $19 \mu \mathrm{M}$ phospholipids vesicles, final concentrations) for $2 \mathrm{~min}$ at $37^{\circ} \mathrm{C}$. After this incubation, a 2- $\mu$ l aliquot was added to $0.3 \mathrm{~nm}$ FXa, $1.5 \mu \mathrm{M}$ prothrombin, and $75 \mu \mathrm{M}$ phospholipids vesicles (0.1 nM FXa, $0.5 \mu \mathrm{M}$ prothrombin, and $25 \mu \mathrm{M}$ phospholipids vesicles, final concentrations) at $37^{\circ} \mathrm{C}$ for $3 \mathrm{~min}$, then stopped with $5 \mu \mathrm{l}$ of ice-cold 0.5 m EDTA. The rate of thrombin substrate cleavage by the consequent reaction mixture was then measured as before. Experiments were performed in triplicate, and data were plotted as the mean residual FVa cofactor activity \pm S.E.

Assessment of APC Variant Binding to Soluble EPCR-APC binding to sEPCR was determined using a BIAcore X100 (GE Healthcare) as previously described $(30,31)$. Briefly, $10 \mu \mathrm{g} / \mathrm{ml}$ monoclonal anti-EPCR antibody, RCR-2 (kind gift of Dr. K. Fukudome, Saga Medical School), was immobilized on to both flow cells of a CM5 sensor chip. sEPCR (31) in HBS-P buffer (100 mм HEPES, pH7.4, $150 \mathrm{~mm} \mathrm{NaCl,} \mathrm{0.005 \%} \mathrm{v/v} \mathrm{surfactant}$ P20) was bound to the surface of the test flow cell (400-800 response units). A reference flow cell with only RCR-2 bound was used to detect nonspecific binding. Wild type or variant APC (25-100 nM) was sequentially injected over both flow cells at a flow rate of $5 \mu \mathrm{l} / \mathrm{min}$ for $60 \mathrm{~s}$. APC-EPCR binding was dissociated using HBS-EP buffer (HBS-P, but containing $3 \mathrm{~mm}$ EDTA; BIAcore) The RCR-2 surface was regenerated with $10 \mu \mathrm{l}$ of $10 \mathrm{~mm}$ glycine- $\mathrm{HCl}$ ( $\mathrm{pH} 2.5$ ) after each experiment.

Measurement of Endothelial Cell Barrier Protection by APCEndothelial cell barrier permeability was determined as described previously with minor modifications (13). Briefly, EAhy926 cells (kind gift of Dr. C. Edgell, University of North Carolina, Chapel Hill, NC) were grown to confluence on polycarbonate membrane transwells (Costar, $3 \mu \mathrm{M}$ pore size, 12-mm diameter) and incubated with $20 \mathrm{~nm}$ wild type or variant APC. After $3 \mathrm{~h}$ the cells were treated with thrombin (Hematologic Technologies) in serum-free media for $10 \mathrm{~min}$. The cells were washed and incubated with $0.67 \mathrm{mg} / \mathrm{ml}$ Evans Blue with $4 \%$ bovine serum albumin (Sigma). Changes in endothelial cell barrier permeability were determined by following the increase in absorbance at $650 \mathrm{~nm}$ in the outer chamber over time due to the transmigration of Evans Blue-bovine serum albumin. Experiments were performed in triplicate and plotted as the mean \pm S.E.

Determination of APC-mediated Protection of Apoptotic Endothelial Cells-Confluent EAhy926 cells in 6-well plates were pretreated with either wild type or variant APC for $17 \mathrm{~h}$. EAhy926 cell apoptosis was induced by staurosporine $(20 \mu \mathrm{M}$,
Sigma) treatment for $4 \mathrm{~h}$. Cells were then trypsinized, and RNA was extracted using the RNeasy Mini kit (Qiagen). Reverse transcription was carried out (High Capacity cDNA reverse transcription kit, Applied Biosystems), then real time PCR was performed using bax (Hs00180269_m1), bcl-2 (Hs00153350_m1) and $\beta$-actin (Hs99999903_m1) Taqman ${ }^{\circledR}$ gene expression assays (Applied Biosystems) in a Applied Biosystems 7500 real time PCR system. Experiments were performed in triplicate and plotted as the mean $b a x / b c l-2$ ratio \pm S.E.

\section{RESULTS}

Characterization of Recombinant Protein C/APC Gla Domain Variants - To identify the critical amino acid residues in the APC Gla domain that mediate protein $\mathrm{S}$ cofactor enhancement of APC anticoagulant activity, recombinant protein C Gla domain variants were generated by site-directed mutagenesis spanning a region of the Gla domain proposed to mediate protein $\mathrm{S}$ cofactor function (Fig. $1 A$ ). Each of these variants (APC-D35T, APC-D36A, APC-L38D, and APC-A39V) contains single amino acid substitutions with the corresponding residue of the human prothrombin Gla domain. This approach enables analysis of APC-specific functions while maintaining Gla domain structural integrity (30). Each expressed recombinant protein $C$ variant was characterized by Western blotting with an anti-protein $\mathrm{C}$ polyclonal antibody and an anti-Gla monoclonal antibody. Recombinant wild type and variant protein $\mathrm{C}$ migrated in a similar fashion to plasmapurified protein $\mathrm{C}$ under non-reducing conditions at the expected molecular mass of $\sim 65 \mathrm{kDa}$. Wild type and variant recombinant protein $C$ exhibited similar band intensity when detected with both anti-protein $\mathrm{C}$ and anti-Gla antibodies to that observed with plasma-purified protein $C$, indicative of normal expression and post-translational modification of the recombinant proteins (Fig. $1 B$ ). For functional studies wild type and variant recombinant protein $C$ were activated using Protac. The amidolytic activity of each recombinant APC variant was assessed and found to be identical to that of wild type APC (Fig. 1C).

The rate of inhibition of each APC variant by serpins was determined in normal plasma. Inhibition of both wild type and variant APC was closely comparable (Fig. $1 D$ ). To examine the role of protein $\mathrm{C}$ inhibitor (PCI), the same experiment was performed in the presence of heparin, which accelerates PCI inhibition of APC. Accordingly, inhibition of wild type APC was enhanced 3-fold, as previously described (32). Each APC variant was inhibited in a similar manner to that of wild type APC in this system (Fig. 1E). Furthermore, subsequent assays indicated that APC inhibition kinetics in the presence of purified $\alpha_{1}$ antitrypsin and protein $C$ inhibitor were not affected by the presence of any of the APC variants tested (data not shown). Therefore, the residue substitutions present in each APC variant do not alter APC inhibition by its known plasma inhibitors.

Determination of Recombinant APC Gla Domain Variant Anticoagulant Activity-To identify the specific APC Gla domain residue(s) responsible for mediating APC anticoagulant activity in plasma, the ability of wild type and variant APC to inhibit tissue factor-induced thrombin generation was 
A
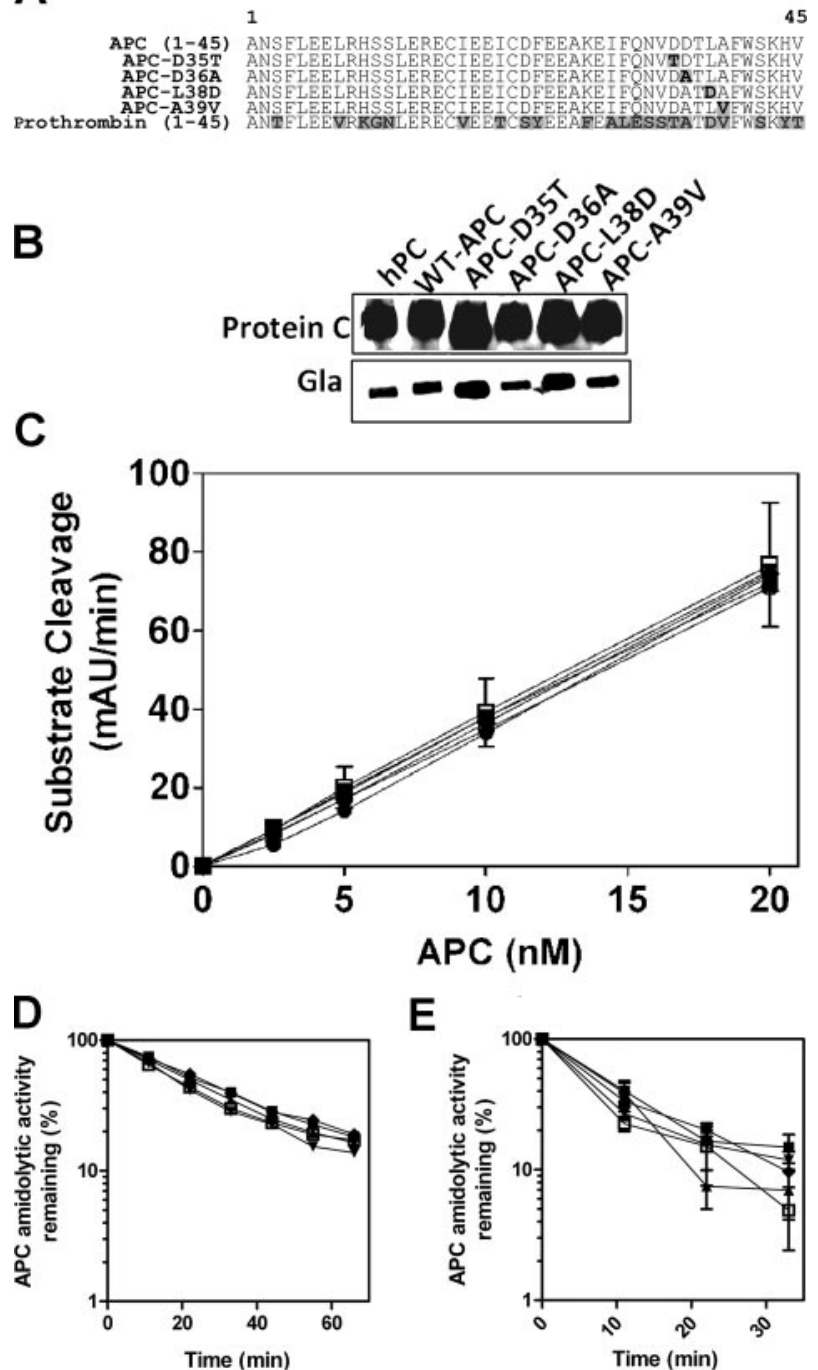

E

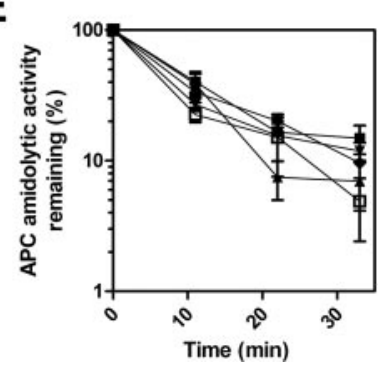

FIGURE 1. Characterization of recombinant APC Gla domain variants. $A$, recombinant protein C Gla domain variants were generated in which protein $C$ residues at positions $35,36,38$, and 39 were replaced with the corresponding residues in human prothrombin. Protein C variants were expressed in HEK 293 cells and then isolated from conditioned serum-free medium. $B$, plasma-purified, recombinant wild type $(W T)$ and variant protein C preparations were assessed by Western blotting with sheep anti-protein $C$ polyclonal and mouse anti-Gla monoclonal antibodies. $h P C$, human PC. $C$, each protein $C$ preparation was activated using Protac, and amidolytic activity of each recombinant APC was assessed using a short APC chromogenic substrate. $m A U$, milliabsorbance units. Inhibition of wild type and variant APC in normal pooled plasma was assessed in the absence $(D)$ and presence $(E)$ of 10 units/ml heparin (wild type, $\square ; A P C-D 35 T, \square ; A P C-D 36 A, \mathbf{\Delta} ; A P C-L 38 D, \nabla ; A P C-$ $A 39 \mathrm{~V}$, ; all $20 \mathrm{~nm})$. Samples were removed at specified time points, and APC amidolytic activity was tested using a chromogenic substrate. The data shown represents the mean of three independent determinations \pm S.D.

assessed. Wild type APC diminished thrombin generation in a concentration-dependent manner, as previously described (30) (Fig. 2A). Variants APC-D35T, APC-D36A, and APC-A39V exhibited mildly impaired anticoagulant activity, with $<2$-fold reduced ETP compared with wild type APC (Fig. 2, $A$ and $B$ ). Variant $A P C-L 38 D$, however, exhibited severely impaired anticoagulant activity (Fig. 2, $A$ and $B$ ). APC-L38D was unable to achieve half-maximal inhibition of thrombin generation (ETP) at concentrations as high as $150 \mathrm{~nm}$ APC (Fig. 2C), compared with half-maximal inhibition observed with wild type APC (7.2
A

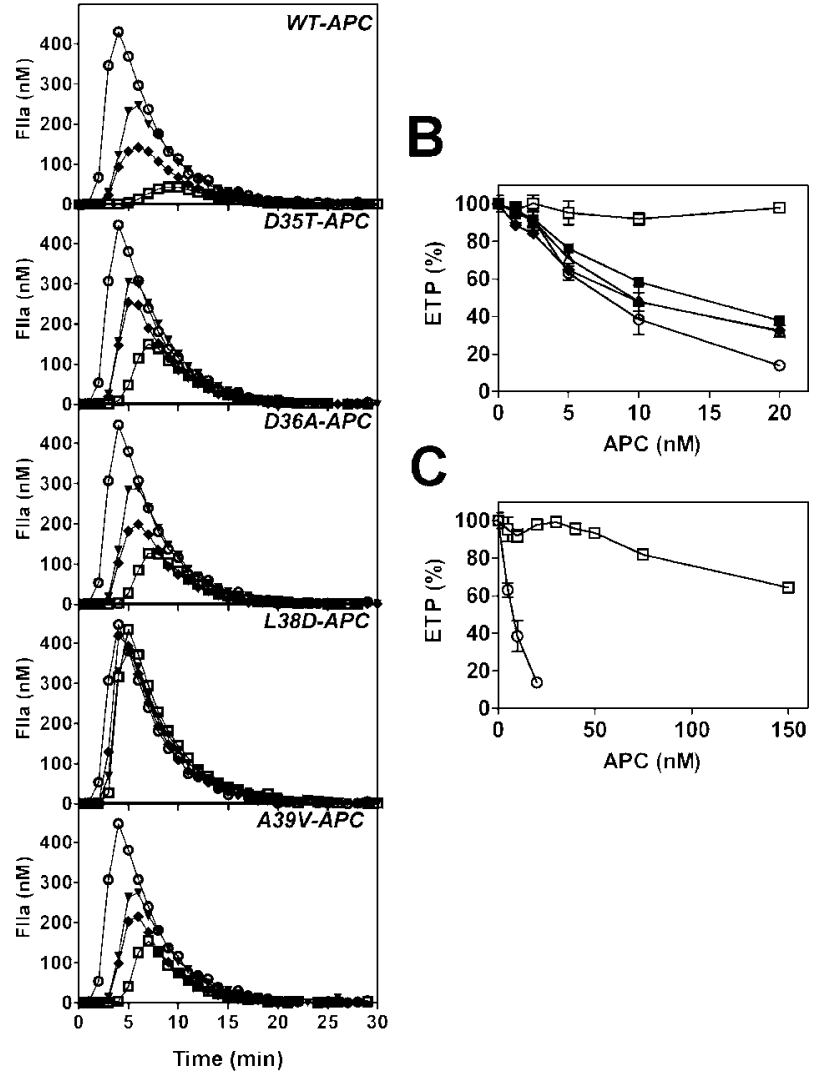

FIGURE 2. Variant APC-L38D has negligible anticoagulant activity in protein C-deficient plasma. The ability of recombinant APC variants to inhibit tissue factor-induced thrombin generation was assessed. A, APC (1.25-20 nM) was incubated with protein C-deficient plasma, and thrombin generation initiated with tissue factor, phospholipid vesicles, and $\mathrm{CaCl}_{2}$ (see "Experimental Procedures"). Thrombin generation in protein C-deficient plasma in the absence of APC $(\bigcirc)$ was compared with $5 \mathrm{nM}(\boldsymbol{\nabla}), 10 \mathrm{nM}(\bullet)$, and $20 \mathrm{nM}(\square)$ of each recombinant APC. $B$, the ETP for each experiment was determined and expressed as \% of thrombin generated in the absence of APC (wild type APC, $(O) ; A P C-D 35 T,(\square) ; A P C-D 36 A, \triangle ; A P C-L 38 D, \square ; A P C-A 39 V, \downarrow$. C, high concentrations (up to $150 \mathrm{nM}$ ) of $A P C-L 38 D(\square)$ was compared with wild type APC (O) in the same assay.

nM; Fig. $2 B)$. These data indicate that $A P C-L 38 D$ is at least 20 -fold less active than wild type APC in plasma.

Leu-38 Is Required for Protein S-dependent APC Anticoagulant Function in Protein S-deficient Plasma-To clarify the role of Leu-38 in facilitating APC anticoagulant function, the ability of APC-L38D to be stimulated in protein S-deficient plasma reconstituted with plasma purified protein $\mathrm{S}$ was determined. Wild type APC had no anticoagulant function in protein S-deficient plasma, as previously observed (33). However, co-incubation of wild type APC with increasing protein S concentrations (12.5-200 nM) caused a corresponding reduction in thrombin generation (ETP) $\left(\mathrm{IC}_{50}=24\right.$ nM protein $\left.\mathrm{S}\right)(\mathrm{Fig} .3, A$ and $B$ ). Variant $A P C-L 38 D$, however, was almost entirely unresponsive to protein $\mathrm{S}$ (Fig. 3, $A$ and $B$ ). At the highest protein $\mathrm{S}$ concentration tested $(1.5 \mu \mathrm{M})$, corresponding to 10 -fold plasma free protein $\mathrm{S}$ concentration, thrombin generation was reduced only $22 \pm 2 \%$ compared with thrombin generated in the absence of protein S. Therefore, residue Leu- 38 mediates APC anticoagulant function in plasma by facilitating critical protein $\mathrm{S}$ cofactor function. 
A
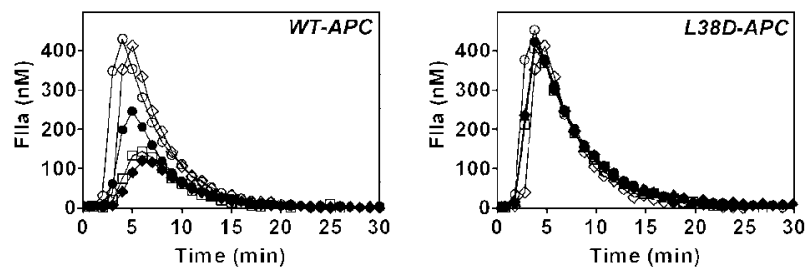

B

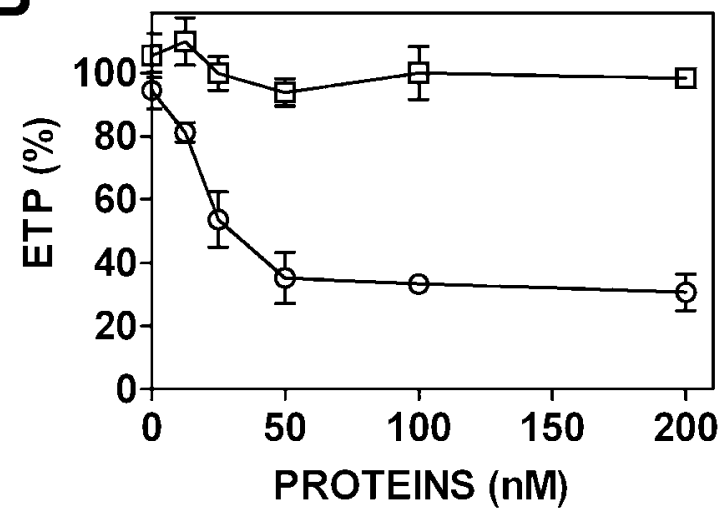

C

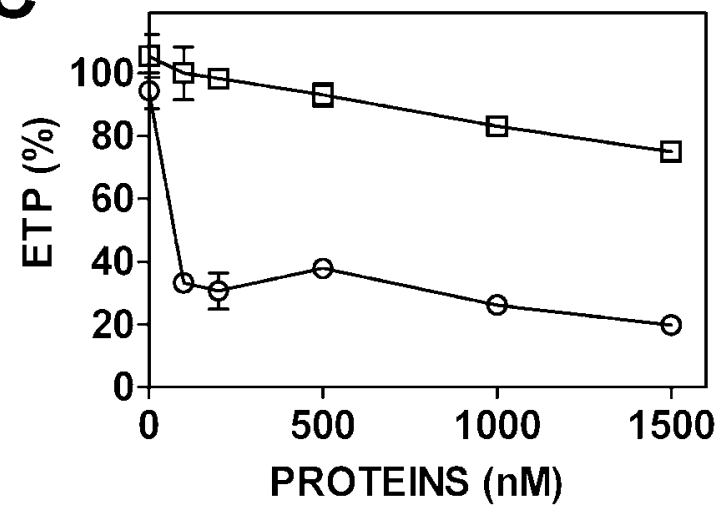

FIGURE 3. Variant APC-L38D is not stimulated by protein S in protein S-deficient plasma. $A$, the ability of recombinant wild type $A P C(O)$ and variant $A P C-L 38 D(\square)$ (both $10 \mathrm{~nm}$ ) to inhibit tissue-factor induced thrombin generation in protein $\mathrm{S}$ deficient plasma was assessed in the presence of increasing plasma purified protein S concentrations; no APC/protein S $(\diamond), 10 \mathrm{~nm}$ APC only (O), APC + $25 \mathrm{~nm}$ protein S (O), APC + $50 \mathrm{~nm}$ protein $\mathrm{S}(\square)$, and APC + 100 $\mathrm{nm}$ protein $\mathrm{S}(\bullet)$. B, the thrombin generation (ETP, \%) in the protein $S$ enhancement of variant APC-L38D $(\square)$ was compared with wild type APC $(\bigcirc)$ in the presence of protein $S$ concentrations $(B)(6-200 \mathrm{~nm})$ and $(C)(0.125-1.5$ $\mu \mathrm{M})$ in the same assay.

APC-L38D Exhibits Normal Protein S-Independent but Severely Impaired Protein S-dependent FVa Proteolysis-To assess the mechanism by which the impaired response to protein $\mathrm{S}$ by $A P C-L 38 D$ in plasma occurs, the rate of FVa proteolysis by APC in the presence and absence of protein $\mathrm{S}$ was determined. Using a phospholipid-dependent FVa proteolysis time course assay, the ability of $A P C-L 38 D$ to inactivate FVa in the absence of protein $\mathrm{S}$ was determined. Both wild type APC and $A P C-L 38 D$ rapidly reduced FVa cofactor activity (Fig. 4, upper panel), indicating that the observed impaired anticoagulant activity in plasma observed for APC-L38D is not mediated by impaired interaction with anionic phospholipids or FVa. A modified version of this assay was used to evaluate FVa proteolysis of wild type and variant APC in response to protein S.
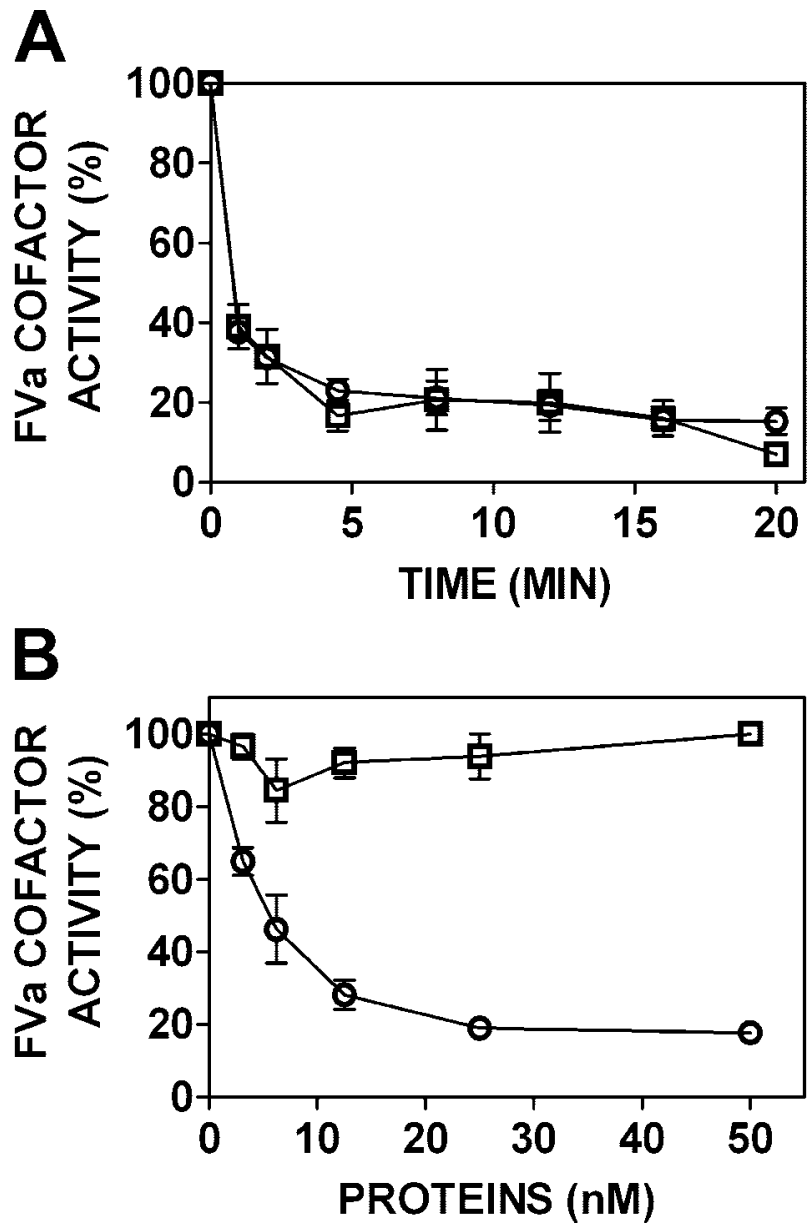

FIGURE 4. Protein S-dependent FVa proteolysis by APC requires residue Leu-38 in the APC Gla domain. Upper panel, FVa (1 nM) was incubated with wild type APC $(\bigcirc)$ or variant APC-L38D $(\square)(0.2 \mathrm{~nm})$ in the presence of $25 \mu \mathrm{M}$ phospholipid vesicles (40\% PC, 20\% PS, 20\% PE). The FVa cofactor activity at specified time points was determined by prothrombinase assay (see "Experimental Procedures"). $B$, human plasma purified protein S (3-50 nM) was incubated with wild type APC $(O)$ or variant $A P C-L 38 D(\square)(0.4 \mathrm{~nm}), \mathrm{FVa}(8 \mathrm{~nm})$, and $25 \mu \mathrm{m}$ phospholipid vesicles (40\% PC, 20\% PS, 20\% PE). After 2 min of incubation, an aliquot was removed and added to a prothrombinase assay to assess FVa cofactor activity.

Wild type APC-mediated FVa proteolysis was rapidly enhanced by protein $\mathrm{S}$ (Fig. 4, lower panel). Half-maximal inhibition of FVa cofactor activity was observed at $5 \mathrm{~nm}$ protein S (Fig. 4, lower panel). In contrast, $A P C-L 38 D$ exhibited no protein S-enhanced FVa proteolysis at each protein $\mathrm{S}$ concentration tested. Therefore, APC-L38D loss of protein S-dependent APC anticoagulant activity observed in plasma occurs via impaired protein S-mediated FVa proteolysis.

APC-L38D Binds sEPCR with Equal Affinity to Wild Type APC-APC-mediated PAR-1 activation is dependent upon APC binding to EPCR. To ascertain whether APC-L38D interacts normally with sEPCR, the binding affinity of this variant compared with wild type APC was tested by surface plasmon resonance. After sEPCR immobilization, wild type APC and $A P C-L 38 D$ (12.5-100 nM) were exposed to the sEPCR surface. $A P C-L 38 D$ bound sEPCR normally $\left(K_{D}=112 \pm 25 \mathrm{~nm}\right)$, similar to that previously described for wild type PC/APC (30, 31) (Fig. 5). 

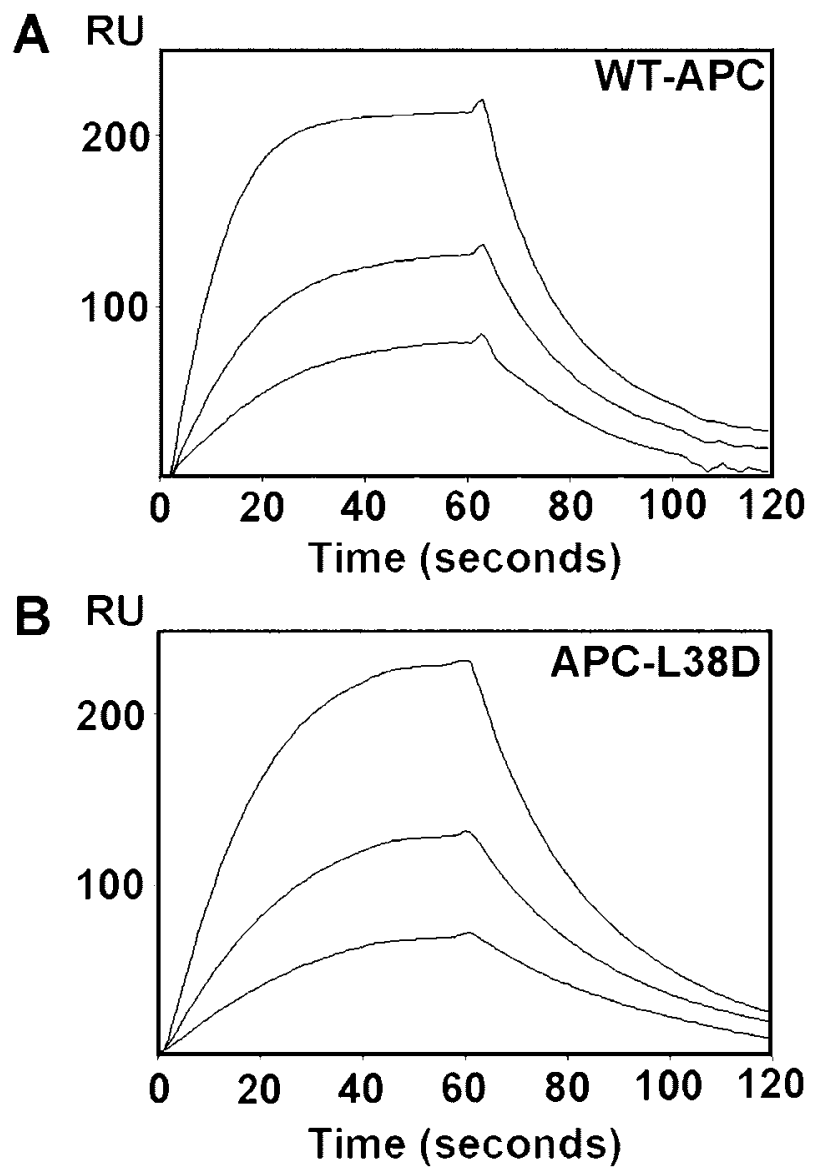

FIGURE 5. APC-L38D has normal affinity for SEPCR. Recombinant APC variant binding to $S E P C R$ was measured by surface plasmon resonance technology. SEPCR (500-600 response units $(R U))$ was immobilized on to the surface of a CM5 sensor chip via a monoclonal anti-EPCR antibody (RCR-2) (see "Experimental Procedures"). An RCR-2 only flow cell was used to detect nonspecific binding. 25-100 nM wild type APC $(A)$ and APC-L38D $(B)$ was passed over the sEPCR surface at a flow rate of $5 \mu \mathrm{l} / \mathrm{min}$. Minor differences in APC levels are due to small differences in SEPCR bound to the sensor chip surface. Binding was assessed using BIAevaluation software package.

APC-L38D Exhibits Identical Anti-inflammatory and Antiapoptotic Activity to Wild Type APC-As APC-L38D bound sEPCR normally, the anti-inflammatory and anti-apoptotic properties of $A P C-L 38 D$ were evaluated. APC can regulate expression of both pro- and anti-apoptotic genes via EPCRmediated PAR-1 signaling (10) to confer a net anti-apoptotic phenotype on endothelial cells. The ability of $A P C-L 38 D$ to protect EAhy 926 cells from apoptosis was assessed by quantification of pro- (bax) and anti- (bcl-2) apoptotic gene expression after incubation with staurosporine, a well characterized inducer of apoptosis in endothelial cells. Staurosporine, as expected, significantly increased (2.3-fold) the $b a x / b c l-2$ ratio compared with untreated EAhy926 cells $(p<0.005)$ (Fig. 6A). Preincubation with either plasma-purified or wild type APC, however, significantly ameliorated the effect of the staurosporine, as indicated by the large reduction in $b a x / b c l-2$ ratio in APC-treated cells $(p<0.005)$ compared with those treated with staurosporine alone. The EPCR dependence of this anti-apoptotic activity was confirmed by incubation of an inhibitory anti-EPCR antibody in conjunction with wild type APC, which led to ablation of the APC-associated protective effect. Of note,
$A P C-L 38 D$ significantly reduced the $b a x / b c l-2$ ratio compared with staurosporine only treated cells $(p<0.005)$ to the same extent as wild type APC (Fig. 6). APC activation of PAR-1 signaling has also previously been described to protect against thrombin-induced endothelial cell barrier hyperpermeability. Using an in vitro assay to assess endothelial cell barrier function, thrombin was found to significantly increase endothelial cell barrier permeability (Fig. 6, $B$ and $C$ ) as previously reported (13). Prior incubation with wild type APC, however, significantly attenuated thrombin-induced endothelial barrier permeability (Fig. 6, $B$ and $C$ ). Each of the APC variants conferred an identical and significant level of endothelial barrier protection to that of wild type APC compared with thrombin-only treated EAhy 926 cells (Fig. 6, $B$ and $C$ ). Therefore, APC variants $A P C-D 35 T, A P C-D 36 A, A P C-L 38 D$, and APC-A39V protect endothelial barrier function to the same extent as wild type APC. These results show that the anti-inflammatory and antiapoptotic functions of APC mediated by EPCR-PAR-1 signaling are entirely conserved in the $A P C-L 38 D$ variant despite significant loss of anticoagulant function caused by the severely impaired response to protein S.

\section{DISCUSSION}

Protein $\mathrm{S}$ is essential for normal anticoagulant function of APC in plasma, acting as a cofactor to enhance proteolysis of APC substrates factor Va and factor VIIIa. Although several putative APC binding sites have been described on protein $S$ (34-40), the corresponding binding sites on APC are poorly defined. However, a previous study has demonstrated that substitution of the APC Gla domain amino acid residues 25-45 with the corresponding amino acid residues in human prothrombin prevented protein S enhancement of APC anticoagulant activity without adversely affecting anionic phospholipid or FVa interactions (41). Furthermore, a subsequent report highlighted the importance of APC Gla domain residues between 33-39, with particular significance assigned to residues Asp-35, Asp-36, Leu-38, and Ala-39 (30).

In this study we have generated and tested individual APC variants in which each of these Gla domain residues was individually substituted with the corresponding prothrombin amino acid residues. Interestingly, only the anticoagulant activity of $A P C-L 38 D$ was severely impaired, exhibiting minimal anticoagulant activity in protein C-deficient plasma compared with wild type APC, even at extremely high concentrations (only $35 \pm 1 \%$ reduction in ETP observed at $150 \mathrm{~nm} A P C-L 38 D$; Fig. 2). Furthermore, when the protein S-dependent anticoagulant activity of this variant was assessed in protein S-deficient plasma (Fig. 3) and in FVa proteolysis assays (Fig. 4), APC-L38D exhibited effectively no response to protein $\mathrm{S}$, even at free protein $\mathrm{S}$ concentrations 10 -fold higher than that found in normal plasma (Fig. 3B). In contrast, the anticoagulant effects of $A P C$ $D 35 T, A P C-D 36 A$, and $A P C-A 39 V$ were only moderately impaired, exhibiting $<2$-fold impaired anticoagulant activity in plasma compared with wild type APC (Fig. 2). These findings suggest that APC Gla domain residues 35,36 , and 39 make a relatively minor contribution to the protein $\mathrm{S}$ interaction with the APC Gla domain in this region. 

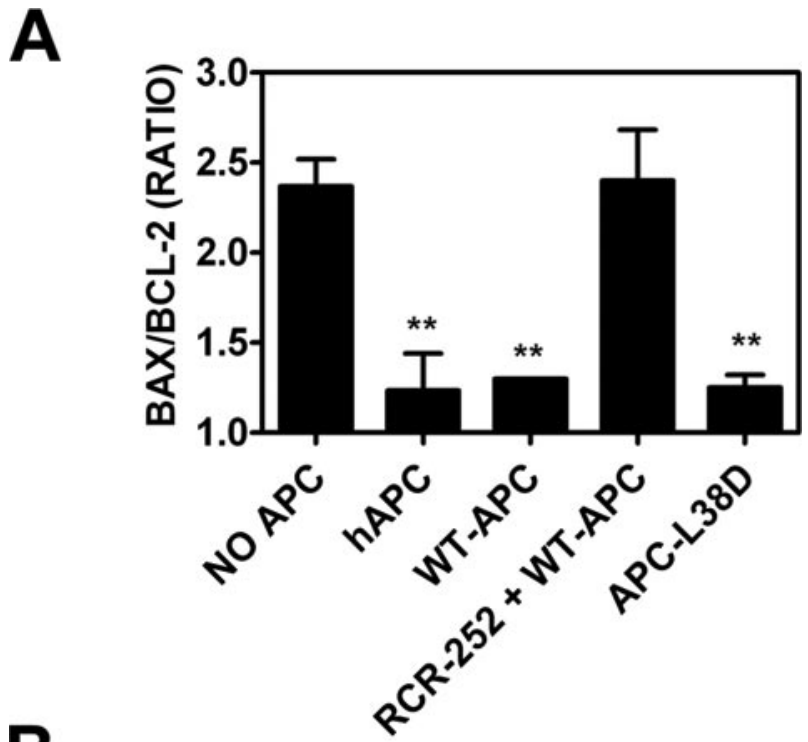

B

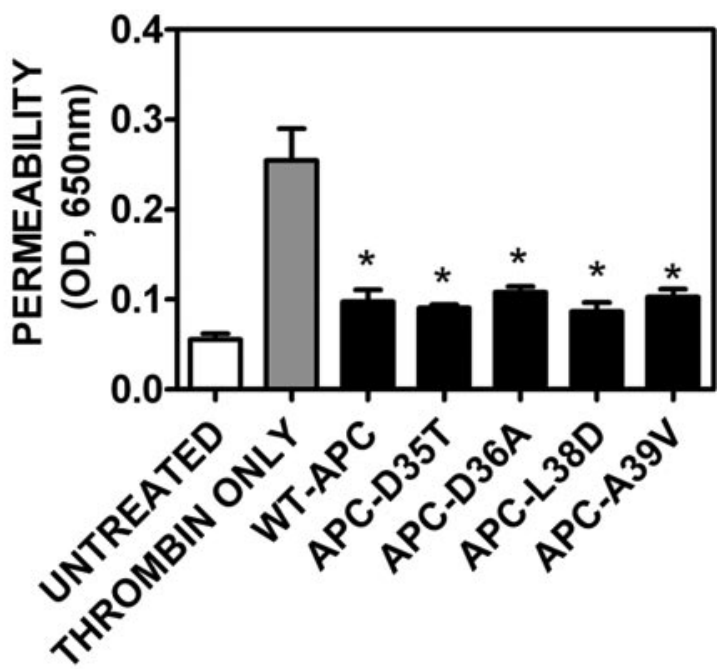

C

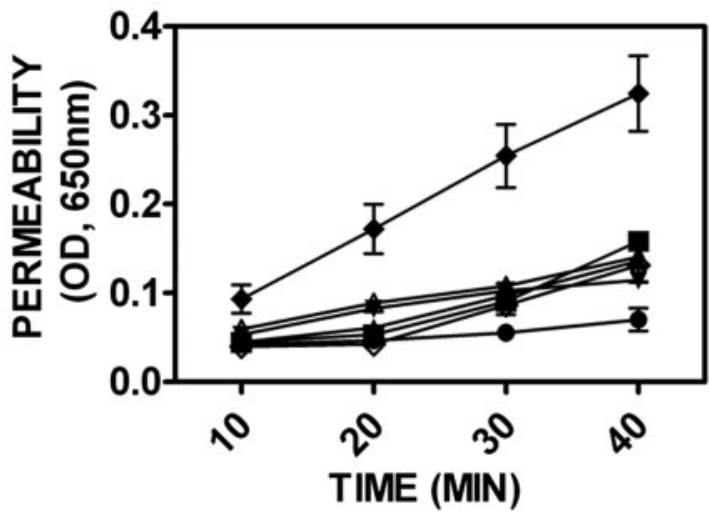

FIGURE 6. APC variants 35-39 exhibit normal ability to inhibit apoptosis and endothelial barrier protection. $A$, confluent EAhy 926 cells were incubated with Protac-generated plasma purified APC, recombinant wild type $A P C$, and APC Gla domain variants for $17 \mathrm{~h}$. A rat monoclonal anti-EPCR antibody (RCR-252) was added $(400 \mu \mathrm{m})$ alongside wild type APC to determine EPCR dependence. Apoptosis was induced in EAhy926 cells by incubation with $20 \mu \mathrm{m}$ staurosporine for $4 \mathrm{~h}$. RNA was extracted and reverse-transcribed as described under "Experimental Procedures." Reverse transcription-PCR was performed using specific $B a x, B C l-2$, and $\beta$-actin primers. Experiments were performed in triplicate, and data are presented as the mean \pm S.E. Unpaired two-tailed $t$ tests were used to determine significance $(* *, p<0.005$ compared with staurosporine-only treated EAhy926 cells). hAPC, human APC.
Protein S binding to APC has historically proved refractory to meaningful assessment in the absence of phospholipid vesicles, making it difficult at this stage to ascertain whether Leu-38 constitutes a key residue as part of a protein $\mathrm{S}$ binding site on APC or is critical for a protein S-mediated conformational change that facilitates enhanced APC substrate proteolysis. Further studies will be required to unravel the precise role of Leu-38 in regulating protein S cofactor enhancement.

Accumulating evidence from different animal disease models suggests that the cytoprotective signaling activity of APC may be of greater significance than its anticoagulant function in protection against various disease states, including severe sepsis, inflammatory bowel disease, and ischemic stroke $(16,19$, 42). Nevertheless, the anticoagulant properties of APC are important in that APC administration is associated with a significant increased bleeding risk (19). Although protein S constitutes a critical cofactor for the anticoagulant function of APC, there is no suggestion that it plays any role in regulating PAR-1-mediated anti-inflammatory or anti-apoptotic effects. Thus, disrupting the interplay between APC and protein S constitutes an attractive and novel strategy that may be exploited to generate APC variants with discrepant anticoagulant to cytoprotective properties. However, previous studies have demonstrated that mutations in other regions of APC may influence the ability of the APC Gla domain to effectively bind EPCR, which is a prerequisite for PAR-1 signaling (24). As we have demonstrated, despite having almost entirely lost the ability to interact with protein $\mathrm{S}$, the $A P C-L 38 D$ variant bound SEPCR with the same affinity as wild type APC (Fig. 5). Furthermore, APC-L38D exhibited PAR-1-mediated cytoprotective properties that were indistinguishable from those of wild type APC (Fig. 6).

Recombinant APC variants possessing APC modifications that have significantly reduced anticoagulant activity but retained significant PAR-1 signaling function have previously been generated using alternative strategies. Mosnier et al. (25) demonstrated that clustered alanine mutations (R229A/ R230A) and (K191A/K192A/K193A) in two surface loops of the APC serine protease domain disrupted the anion binding site on APC for FVa exosite binding. Consequently, when these five alanine substitutions were combined (5A-APC), APC anticoagulant activity was markedly reduced $(<3 \%$ residual APTT clotting assay) (26). Because the APC serine protease exosite for PAR-1 is distinct from that of FVa, this combination of mutations did not influence the cytoprotective properties of 5A-APC, which were identical to those of wild type APC. Thus the anticoagulant and anti-inflammatory profiles of this $5 \mathrm{~A}-\mathrm{APC}$ variant are comparable with that exhibited by the

$B$, the protective effect of APC on the endothelial cell barrier was determined for wild type APC and each Gla domain variant. EAhy926 cells were preincubated with $20 \mathrm{~nm}$ wild type or variant APC (black bars) for $3 \mathrm{~h}$. Untreated cells (white bar) were used as a negative control. EAhy926 cells were then treated with $5 \mathrm{~nm}$ thrombin in serum-free media for $10 \mathrm{~min}$ (cells treated with thrombin only, gray bar), and endothelial barrier permeability was assessed after 30 min using Evans Blue-bovine serum albumin (see "Experimental Procedures"). Unpaired two-tailed $t$ tests were used to determine significance $(*$, $p<0.05$ compared with thrombin-only treated EAhy 926 cells). $C$, endothelial barrier protection was assessed over time as described above: untreated EAhy926 cells, $5 \mathrm{~nm}$ thrombin, ; WT-APC, $\Delta ; A P C-D 35 T, \square ; A P C-D 36 A, \triangle$; APC-L38D, $\diamond ; A P C-A 39 \mathrm{~V}, \mathbf{\nabla}$. 
$A P C-L 38 D$ variant described herein despite the fact that the respective mutations occur at opposite ends of the APC molecule, are located in distinct structural domains, and target two different specific APC functional interactions.

Using an alternative approach, Bae et al. (24) engineered a disulfide bond (Cys ${ }^{67}-\mathrm{Cys}^{82}$; chymotrypsin numbering) within the APC serine protease domain, which prevented $\mathrm{Ca}^{2+}$ binding to the functionally critical $\mathrm{Ca}^{2+}$ binding 70-80 loop. As a result, the anticoagulant activity of the variant $\mathrm{Cys}^{67}$ Cys ${ }^{82}$ APC was again dramatically impaired, but it was shown to retain PAR-1 activation mediated anti-apoptotic, anti-inflammatory, and endothelial barrier protective functions. However, in contrast to $5 \mathrm{~A}-\mathrm{APC}$ and the APC-L38D variant, the cytoprotective effects of $\mathrm{Cys}^{67}-\mathrm{Cys}^{82}-\mathrm{APC}$ required a 2 -fold higher concentration of mutant $\mathrm{APC}$ to produce equivalent effects to wild type APC. Interestingly this reduction in the cytoprotective properties of $\mathrm{Cys}^{67}-\mathrm{Cys}^{82} \mathrm{APC}$ was subsequently shown to result from a reduced affinity of the variant APC for EPCR.

On the basis of their specifically reduced anticoagulant activity, one would anticipate that these two previously described recombinant APC variants together with the novel APC-L38D variant should all be associated with significantly reduced bleeding risk in vivo. Because of their reduced bleeding risk, these variants may permit the use of significantly higher APC doses and also longer duration APC administration. Such altered APC therapeutic regimens may be particularly relevant in severe sepsis where overall mortality in the group of patients treated with wild type APC in the PROWESS trial still exceeded $24 \%$ (19). A recent in vivo study has confirmed for the first time that APC variants with reduced or minimal anticoagulant activity may confer important therapeutic benefit. In a murine model, Kerschen et al. (8) demonstrate that 5A-APC was as effective as wild type APC in reducing overall mortality after LPS challenge.

In addition to the complete ablation of anticoagulant activity for $A P C-L 38 D$, we observed a 2-fold reduced anticoagulant activity for each of the variants $A P C-D 35 T, A P C-D 36 A$, and $A P C-A 39 V$ respectively. Each of these variants also retained entirely normal PAR-1-mediated cytoprotective properties. Thus, these three variants demonstrate anticoagulant/cytoprotective phenotypes that are intermediate between wild type APC and the APC-L38D variant. Consequently, these variants may prove useful adjuncts for defining the relative importance of the anticoagulant and cytoprotective effects of APC in different disease states. Moreover, such APC variants with reduced but residual anticoagulant activity may also offer novel therapeutic opportunities. For example, a beneficial effect of APC has recently been described in a murine model of experimental autoimmune encephalomyelitis (17). In this animal model of multiple sclerosis, it was clearly demonstrated that both the cytoprotective and the anticoagulant properties of APC were required for maximal therapeutic efficacy. These findings suggest that second generation therapeutic recombinant APC variants that retain some residual anticoagulant activity may constitute the treatment of choice in specific disease settings. Further in vivo studies will be necessary to fully dissect the relative contributions of APC anticoagulant versus cytoprotec- tive properties across such a wide variety of pathophysiological processes.

In conclusion, we have demonstrated that a single amino acid substitution (L38D) in the Gla domain of APC is sufficient to almost entirely ablate APC anticoagulant activity due to severely impaired protein $\mathrm{S}$ cofactor response. This variant, however, retains normal EPCR binding and PAR-1 signaling properties, suggesting a novel mechanism by which the anticoagulant and cytoprotective properties of APC can be separated for potential therapeutic gain.

\section{REFERENCES}

1. Esmon, C. T. (1989) J. Biol. Chem. 264, 4743-4746

2. Stearns-Kurosawa, D. J., Kurosawa, S., Mollica, J. S., Ferrell, G. L., and Esmon, C. T. (1996) Proc. Natl. Acad. Sci. U. S. A. 93, 10212-10216

3. Walker, F. J., Sexton, P. W., and Esmon, C. T. (1979) Biochim. Biophys. Acta 571, 333-342

4. Fay, P. J., Smudzin, T. M., and Walker, F. J. (1991) J. Biol. Chem. 266, 20139-20145

5. Seligsohn, U., Berger, A., Abend, M., Rubin, L., Attias, D., Zivelin, A., and Rapaport, S. I. (1984) N. Engl. J. Med. 310, 559-562

6. Reitsma, P. H., Poort, S. R., Bernardi, F., Gandrille, S., Long, G. L., Sala, N., and Cooper, D. N. (1993) Thromb. Haemostasis 69, 77-84

7. Riewald, M., Petrovan, R. J., Donner, A., Mueller, B. M., and Ruf, W. (2002) Science 296, $1880-1882$

8. Kerschen, E. J., Fernandez, J. A., Cooley, B. C., Yang, X. V., Sood, R., Mosnier, L. O., Castellino, F. J., Mackman, N., Griffin, J. H., and Weiler, H. (2007) J. Exp. Med. 204, 2439-2448

9. Joyce, D. E., Gelbert, L., Ciaccia, A., DeHoff, B., and Grinnell, B. W. (2001) J. Biol. Chem. 276, 11199-11203

10. Riewald, M., and Ruf, W. (2005) J. Biol. Chem. 280, 19808-19814

11. White, B., Schmidt, M., Murphy, C., Livingstone, W., O’Toole, D., Lawler, M., O’Neill, L., Kelleher, D., Schwarz, H. P., and Smith, O. P. (2000) Br. J. Haematol. 110, 130-134

12. Feistritzer, C., Lenta, R., and Riewald, M. (2005) J. Thromb. Haemost. 3, 2798-2805

13. Feistritzer, C., and Riewald, M. (2005) Blood 105, 3178-3184

14. Domotor, E., Benzakour, O., Griffin, J. H., Yule, D., Fukudome, K., and Zlokovic, B. V. (2003) Blood 101, 4797-4801

15. Cheng, T., Liu, D., Griffin, J. H., Fernandez, J. A., Castellino, F., Rosen, E. D., Fukudome, K., and Zlokovic, B. V. (2003) Nat. Med. 9, 338-342

16. Griffin, J. H., Zlokovic, B., and Fernandez, J. A. (2002) Semin. Hematol. 39, 197-205

17. Han, M. H., Hwang, S. I., Roy, D. B., Lundgren, D. H., Price, J. V., Ousman, S. S., Fernald, G. H., Gerlitz, B., Robinson, W. H., Baranzini, S. E., Grinnell, B. W., Raine, C. S., Sobel, R. A., Han, D. K., and Steinman, L. (2008) Nature 451, 1076-1081

18. Isermann, B., Vinnikov, I. A., Madhusudhan, T., Herzog, S., Kashif, M., Blautzik, J., Corat, M. A., Zeier, M., Blessing, E., Oh, J., Gerlitz, B., Berg, D. T., Grinnell, B. W., Chavakis, T., Esmon, C. T., Weiler, H., Bierhaus, A., and Nawroth, P. P. (2007) Nat. Med. 13, 1349-1358

19. Bernard, G. R., Vincent, J. L., Laterre, P. F., LaRosa, S. P., Dhainaut, J. F., Lopez-Rodriguez, A., Steingrub, J. S., Garber, G. E., Helterbrand, J. D., Ely, E. W., and Fisher, C. J., Jr. (2001) N. Engl. J. Med. 344, 699-709

20. Warren, B. L., Eid, A., Singer, P., Pillay, S. S., Carl, P., Novak, I., Chalupa, P., Atherstone, A., Penzes, I., Kubler, A., Knaub, S., Keinecke, H. O., Heinrichs, H., Schindel, F., Juers, M., Bone, R. C., and Opal, S. M. (2001) J. Am. Med. Assoc. 286, 1869-1878

21. Abraham, E., Reinhart, K., Opal, S., Demeyer, I., Doig, C., Rodriguez, A. L., Beale, R., Svoboda, P., Laterre, P. F., Simon, S., Light, B., Spapen, H., Stone, J., Seibert, A., Peckelsen, C., De Deyne, C., Postier, R., Pettila, V., Artigas, A., Percell, S. R., Shu, V., Zwingelstein, C., Tobias, J., Poole, L., Stolzenbach, J. C., and Creasey, A. A. (2003) J. Am. Med. Assoc. 290, $238-247$

22. Bertolini, G., Rossi, C., Anghileri, A., Livigni, S., Addis, A., and Poole, D. (2007) Intensive Care Med. 33, 426-434. 
Protein S Enhancement of APC Anticoagulant Function

23. Kanji, S., Perreault, M. M., Chant, C., Williamson, D., and Burry, L. (2007) Intensive Care Med. 33, 517-523

24. Bae, J. S., Yang, L., Manithody, C., and Rezaie, A. R. (2007) J. Biol. Chem. 282, 9251-9259

25. Mosnier, L. O., Gale, A. J., Yegneswaran, S., and Griffin, J. H. (2004) Blood 104, $1740-1744$

26. Mosnier, L. O., Yang, X. V., and Griffin, J. H. (2007) J. Biol. Chem. 282, 33022-33033

27. Walker, F. J. (1984) Semin. Thromb. Hemostasis 10, 131-138

28. Yegneswaran, S., Smirnov, M. D., Safa, O., Esmon, N. L., Esmon, C. T., and Johnson, A. E. (1999) J. Biol. Chem. 274, 5462-5468

29. Nicolaes, G. A., Tans, G., Thomassen, M. C., Hemker, H. C., Pabinger, I., Varadi, K., Schwarz, H. P., and Rosing, J. (1995) J. Biol. Chem. 270, 21158-21166

30. Preston, R. J., Ajzner, E., Razzari, C., Karageorgi, S., Dua, S., Dahlback, B., and Lane, D. A. (2006) J. Biol. Chem. 281, 28850-28857

31. Preston, R. J., Villegas-Mendez, A., Sun, Y. H., Hermida, J., Simioni, P., Philippou, H., Dahlback, B., and Lane, D. A. (2005) FEBS J. 272, 97-108

32. Berg, D. T., Gerlitz, B., Shang, J., Smith, T., Santa, P., Richardson, M. A., Kurz, K. D., Grinnell, B. W., Mace, K., and Jones, B. E. (2003) Proc. Natl. Acad. Sci. U. S. A. 100, 4423-4428
33. Hackeng, T. M., Sere, K. M., Tans, G., and Rosing, J. (2006) Proc. Natl. Acad. Sci. U. S. A. 103, 3106-3111

34. Dahlback, B., Hildebrand, B., and Malm, J. (1990) J. Biol. Chem. 265, 8127-8135

35. Giri, T. K., de Frutos, P. G., Yamazaki, T., Villoutreix, B. O., and Dahlback, B. (1999) Thromb. Haemostasis 82, 1627-1633

36. He, X., Shen, L., and Dahlback, B. (1995) Eur. J. Biochem. 227, 433-440

37. He, X., Shen, L., Villoutreix, B. O., and Dahlback, B. (1998) J. Biol. Chem. 273, 27449-27458

38. Mille-Baker, B., Rezende, S. M., Simmonds, R. E., Mason, P. J., Lane, D. A., and Laffan, M. A. (2003) Blood 101, 1416-1418

39. Saller, F., Kaabache, T., Aiach, M., Gandrille, S., and Borgel, D. (2006) J. Thromb. Haemost. 4, 704-706

40. Saller, F., Villoutreix, B. O., Amelot, A., Kaabache, T., Le Bonniec, B. F., Aiach, M., Gandrille, S., and Borgel, D. (2005) Blood 105, 122-130

41. Smirnov, M. D., Safa, O., Regan, L., Mather, T., Stearns-Kurosawa, D. J., Kurosawa, S., Rezaie, A. R., Esmon, N. L., and Esmon, C. T. (1998) J. Biol. Chem. 273, 9031-9040

42. Scaldaferri, F., Sans, M., Vetrano, S., Graziani, C., De Cristofaro, R., Gerlitz, B., Repici, A., Arena, V., Malesci, A., Panes, J., Grinnell, B. W., and Danese, S. (2007) J. Clin. Investig. 117, 1951-1960 